\title{
Adenoid Cystic Carcinoma of the Breast: A Case Series of Six Patients and Literature Review
}

\author{
Miso Kim, MD ${ }^{1}$ \\ Dae-Won Lee, MD' \\ Jin Im, MD \\ Koung Jin Suh, MD 1 \\ Bhumsuk Keam, MD \\ Hyeong-Gon Moon, MD, PhD² \\ Seock-Ah Im, MD PhD' \\ Wonshik Han, MD, PhD² \\ In Ae Park, MD, PhD ${ }^{3}$ \\ Dong-Young Noh, MD, PhD²
}

Departments of ${ }^{1}$ Internal Medicine, ${ }^{2}$ Surgery, and ${ }^{3}$ Pathology,

Seoul National University Hospital,

Seoul National University

College of Medicine, Seoul, Korea
Adenoid cystic carcinoma (ACC) of the breast is a very rare and indolent tumor with a favorable prognosis, despite its triple-negative status. Due to its rarity, there has been no consensus regarding treatments, and treatment guidelines have not been established. Here, we report on six patients with ACC of the breast. All of the patients initially presented with localized disease and no axillary lymph node metastases. Although some of our patients developed local recurrence or distant metastases, all patients had a favorable clinical course, and to date, none of the patients has died from complications of her disease. Here, we described the clinicopathologic features of ACC of the breast and review the current literature.

\section{Key words}

Adenoid cystic carcinoma, Breast neoplasms,

Triple-negative breast carcinoma

Correspondence: Bhumsuk Keam, MD

Department of Internal Medicine,

Seoul National University Hospital,

Seoul National University College of Medicine,

101 Daehak-ro, Jongno-gu, Seoul 110-744, Korea

Tel: 82-2-2072-7215

Fax: 82-2-2072-7379

E-mail: bhumsuk@snu.ac.kr

Received February 10, 2013

Accepted March 15, 2013

\section{Introduction}

Adenoid cystic carcinoma (ACC) of the breast is a rare type of primary breast cancer accounting for less than $0.1 \%$ of all primary breast cancers [1]. ACC of the breast, previously called "cylindroma", was first described by Geschickter and Copeland in 1945 [2]. The majority of ACCs arising in the breast have been localized disease with a low rate of axillary lymph node involvement and distant metastases. ACCs of the breast have favorable outcomes compared with other forms of breast cancer and ACCs of the salivary glands [3]. The 10-year survival rate for patients with ACCs of the breast has ranged from $85 \%$ to $100 \%$ [4,5]. Due to the rarity of the tumor and large variations in the patterns of practice, guidelines for treatment have not been established.

This aim of this report is to present our experience with patients with ACC of the breast and to review the clinicopathologic features and current preferred treatment modalities. 


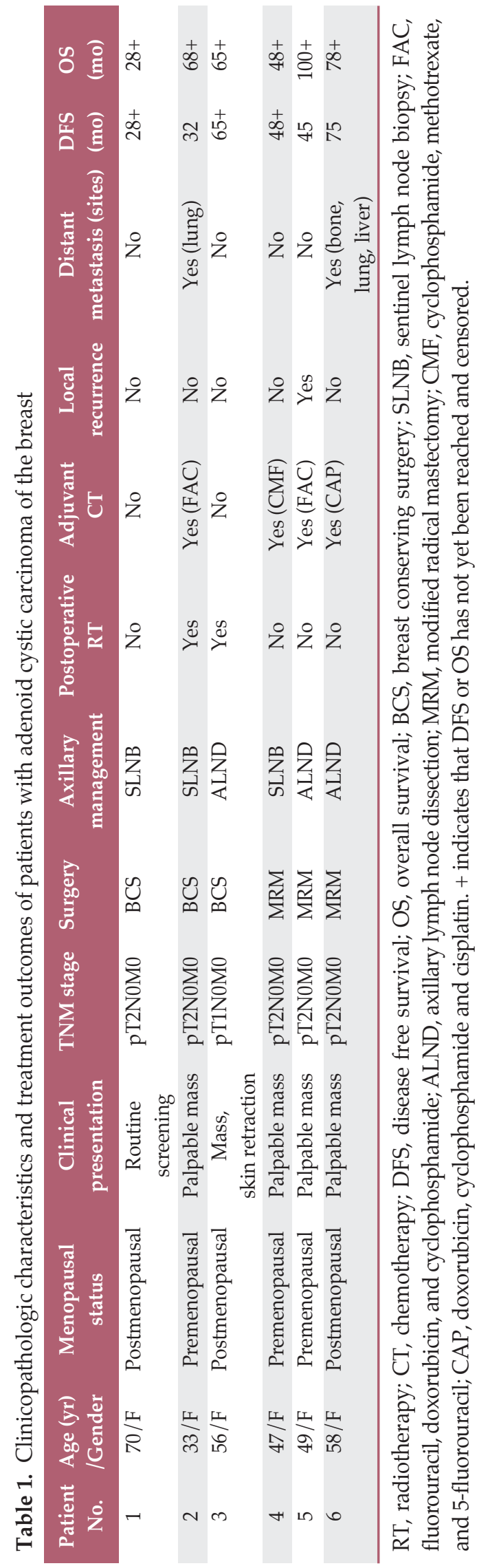

\section{Case Reports}

The medical records of six patients diagnosed with ACC of the breast between March 2004 and June 2010 were retrospectively reviewed. A summary of the clinicopathologic characteristics of the tumors and patient's treatments is shown in Table 1.

\section{Case 1}

A 70-year-old female had a mass in her left breast, which was detected on a routine screening mammogram. Mammography and breast ultrasonography (US) showed an irregular mass measuring $2.4 \times 1.4 \mathrm{~cm}$ with a spiculated margin in the upper inner quadrant. Breast magnetic resonance imaging (MRI) showed an irregular enhancing mass in the left breast. Results of a subsequent diagnostic core biopsy indicated an infiltrative ductal carcinoma. The patient underwent quadrantectomy with sentinel lymph node biopsy (SLNB). The tumor was found to be an ACC measuring $2.0 \times 1.9 \times 1.5 \mathrm{~cm}$ (nuclear grade, 2 ), and the sentinel lymph node was negative for metastasis (pT2N0M0). Immunohistochemistry (IHC) of the tumor was negative for estrogen receptor (ER), progesterone receptor (PR), and human epidermal growth factor receptor 2 (HER2) expression. The patient did not receive adjuvant radiotherapy or chemotherapy. The patient has been free of recurrence during 28 months of follow-up.

\section{Case 2}

A 33-year-old female presented with a mass in her right breast, which was discovered during self-examination. The patient underwent a right inner quadrantectomy with SLNB. Histopathologic examination of the tumor specimen after surgery showed an ACC of low nuclear grade measuring $1.6 \times 1.2 \times 2.5 \mathrm{~cm}$ and no metastasis to the sentinel lymph node (pT2N0M0). The tumor was negative for expression of ER, PR, and HER2. The patient subsequently received adjuvant chemotherapy with fluorouracil, doxorubicin, and cyclophosphamide (FAC), followed by adjuvant radiation therapy. After 28 disease-free months, chest computed tomography (CT) showed multiple lung nodules that were suspicious for metastatic disease. The patient underwent pulmonary metastasectomy using wedge resection of the left upper and lower lobes. Histopathology indicated metastatic ACC. The largest of eight metastases measured $0.9 \mathrm{~cm}$. The patient received first-line palliative chemotherapy with docetaxel every three weeks. After six cycles, response assessment showed stable disease, and chemotherapy was discontinued. 
Seventeen months later, chest CT showed that the lung nodules had become larger. Because of her poor overall condition, the patient received three cycles of capecitabine without further chemotherapy. She has had stable disease for the past 12 months.

\section{Case 3}

A 56-year-old female was found to have a palpable mass and skin retraction involving the left breast. Mammography and breast US showed a conglomerate mass measuring 2.6 $\mathrm{cm}$ and a daughter nodule measuring $0.5 \mathrm{~cm}$ in the lower inner quadrant. Breast MRI showed two intensely enhancing round and irregular masses and skin retraction. Left quadrantectomy and axillary lymph node dissection (ALND) were performed. The tumors were ACCs with low nuclear grade, measuring $1.4 \times 1.2 \times 1.2 \mathrm{~cm}$ and $1.2 \times 1.1 \times 0.9 \mathrm{~cm}$. No metastatic axillary lymph nodes were observed (pT1N0M0). IHC was negative for ER, PR, and HER2 expression. The patient subsequently received adjuvant radiation therapy. She has been free of recurrence for 65 months.

\section{Case 4}

A 47-year-old female presented with a palpable mass in her left breast, which she had discovered three years before being seen by us. Mammography and breast US showed multiple masses in the left upper breast. Breast MRI showed an enhancing lobulated mass measuring $1.1 \times 1.2 \mathrm{~cm}$ in the left upper breast and multiple small adjacent and posterior nodules. Results of a subsequent diagnostic core biopsy revealed an infiltrative ductal carcinoma with features of ACC. The patient underwent a modified radical mastectomy (MRM) with SLNB. Histopathologic examination of the resected specimen showed multiple ACCs measuring a total of $4.2 \times 2.3 \times 3 \mathrm{~cm}$. There were no metastatic lymph nodes (pT2N0M0). IHC was negative for ER, PR, and HER2 expression. The patient underwent six cycles of adjuvant chemotherapy using cyclophosphamide, methotrexate, and 5 -fluorouracil. She has been free of recurrence for 48 months.

\section{Case 5}

A 49-year-old female presented with a palpable mass in her right breast, which she had discovered one year earlier. Mammography and breast US showed a poorly defined mass measuring $3.0 \times 2.5 \mathrm{~cm}$ in the right upper breast and thickening of the overlying skin. The patient underwent MRM and ALND. Histopathologic examination revealed an ACC measuring $4.5 \times 3.7 \times 2.5 \mathrm{~cm}$ and no metastatic lymph nodes (pT2N0M0). The tumor consisted of $50 \%$ solid component in addition to necrosis and infiltrative invasion. IHC was negative for expression of ER, PR, and HER2. The patient underwent six cycles of adjuvant chemotherapy using FAC. She was free of disease for 45 months, and was then seen with thickened, reddish skin at the previous surgical site. Results of a skin biopsy indicated recurrent ACC. Local excision was performed, followed by a wide excision and postoperative radiotherapy, because the posterior and lateral resection margins were involved with the tumor. No recurrence has been detected during 52 months of follow-up.

\section{Case 6}

A 58-year-old femalewith ACC of the right breast had undergone right MRM and ALND followed by six cycles of adjuvant chemotherapy using doxorubicin, cyclophosphamide, and cisplatin. Six years later, she presented with a mass in her right scapula of two months duration. Shoulder MRI showed a large osteolytic mass involving the right scapula. ${ }^{18} \mathrm{~F}$-fluoro-2-D-glucose positron emission tomography/computed tomography $\left({ }^{18} \mathrm{FDG}-\mathrm{PET} / \mathrm{CT}\right)$ showed multiple hypermetabolic lesions in the lungs, bones, and liver. Histopathologic examination of the earlier ACC specimen revealed a tumor with high nuclear grade. Percutaneous biopsy of the scapular tumor was performed using a biopsy gun, and the histopathologic findings were similar to those of the previous mastectomy specimen. IHC of the tumor cells was negative for ER and PR expression. Fluorescent in-situ hybridization analysis showed a negative result for amplification of the HER2 / neu gene. The patient was diagnosed with recurrent ACC, with metastases to the lung, bone, and liver. She received palliative radiotherapy for the scapular metastases, and was enrolled in a clinical trial. One month after the patient began treatment, a follow-up CT scan and ${ }^{18} \mathrm{FDG}$-PET/CT showed disease progression.

\section{Discussion}

ACC of the breast is a very rare malignancy, accounting for less than $0.1 \%$ of all breast neoplasms [1]. Most information on ACC of the breast is derived from individual case reports and clinical case series. Only one Korean patient with ACC of the breast has been reported in the literature [6]. According to findings of a recent large population-based cohort study conducted by Ghabach et al. [7], which included 338 patients in the United States, the incidence of ACC of the breast has remained stable over a 30-year period (1977-2006).

A palpable breast mass is the most common presenting 
symptom of breast ACC; a few cases have also been detected incidentally on routine screening mammograms of asymptomatic patients [8]. The radiologic appearance is generally nonspecific, and little has been published on the mammographic and ultrasonographic features of ACCs [9].

The histopathologic features of ACC of the breast are similar to those of ACC originating from salivary glands and are distinct from those of ductal and lobular carcinomas of the breast; however, the histopathologic appearance of ACC may resemble that of invasive ductal carcinoma [10]. The histopathology of the core biopsy samples from two of our patients was thought to be infiltrating ductal carcinoma, whereas ACC was the final pathologic diagnosis of the surgically resected specimens. ACC is generally negative for ER and PR expression and does not show HER2/neu gene amplification. The epidermal growth factor receptor (EGFR) protein is commonly expressed in the absence of EGFR gene alterations, and EGFR is potentially an important therapeutic target [11]. In addition, several studies have reported that markers with stem cell-like features, such as c-KIT, are commonly expressed in ACC [11]. Although triple-negative breast cancer (TNBC) generally has high proliferative activity, several studies using proliferative markers (e.g., Ki-67) reported a low proliferation rate in ACC of the breast $[11,12]$. It is probable that specific molecular characteristics of ACC of the breast affect its more indolent clinical course and favorable prognosis, compared to TNBC with basal-like features.

Treatment for ACC of the breast is similar to treatment for ACC of the salivary glands. The standard treatment for ACC of the salivary glands is surgical excision with wide margins usually followed by postoperative radiotherapy, because ACC of the salivary glands manifests locally aggressive behavior and frequently shows local recurrence [13]. Currently, there is little evidence indicating that adjuvant chemotherapy has improved the outcomes of patients with ACC of the salivary glands. The lung is the most common site of metastasis.

There is no consensus on the optimal management for patients with ACC of the breast. Treatment has been mainly surgical, ranging from local excision to radical mastectomy. Guidelines for the extent of resection have not yet been established. Three patients in our series underwent breast conserving surgery and three patients underwent MRM. Leeming et al. [14], who evaluated 24 cases, found that $37.5 \%$ of patients had local recurrence of ACC after undergoing local excision only. Although the relationship between margin status and recurrence may not be clear, local excision alone may result in unacceptably high rates of local recurrence. Due to the low rate of axillary lymph node metastasis, the role of ALND for patients with ACC remains unclear. ALND was performed for three of our patients, and three patients underwent SLNB. All six patients had negative lymph nodes.

Several retrospective studies have demonstrated that adjuvant radiotherapy may be effective for local control and survival in patients with ACC of the breast $[4,15]$. A recent large retrospective study that included 376 patients with ACC of the breast identified by the Surveillance, Epidemiology, and End Results (SEER) database found that adjuvant radiotherapy after local surgical therapy improved both overall survival and disease-specific survival [15]. Indications for use of adjuvant radiotherapy for patients have not yet been determined.

Due to insufficient data, the value of systemic adjuvant chemotherapy for ACC of the breast has not been established. Two of our patients received adjuvant radiotherapy and four patients received adjuvant chemotherapy at the discretion of their treating physician. The decision to administer adjuvant therapy was based on current evidence indicating that adjuvant systemic treatment for classic types of breast cancer is advantageous. In our patient cohort, local recurrence occurred in one patient who had undergone MRM and ALND followed by systemic adjuvant chemotherapy.

Distant metastases from ACC of the breast are uncommon, and they tend to occur without lymph node involvement [14]. The most frequent site of metastasis is lung; other sites include bone, liver, and kidney, which are also the metastatic sites in patients with ACC of the salivary glands [5]. Distant metastasis to the lung and bone occurred in two of our patients. Although systemic chemotherapy for metastatic ACC of the breast appeared to have little efficacy for our patients, the metastases were slow-growing tumors. Although some of our patients had local recurrence or distant metastases, all patients had an indolent clinical course; and to date, none of our patients has died of complications of her disease.

ACCs of the breast are rare triple-negative tumors. Although ACCs of the breast generally have a favorable clinical course, distant metastasis and late recurrence can arise, and long term follow-up is required. Currently, there is no consensus on the optimal treatment modalities; further assessment of the roles of breast preserving surgery, ALND, and adjuvant treatment is needed. Additional clinical experience is needed in order to establish the optimal guidelines for treatment of ACC of the breast.

\section{Conflicts of Interest}

Conflict of interest relevant to this article was not reported. 


\section{References}

1. Muslimani AA, Ahluwalia MS, Clark CT, Daw HA. Primary adenoid cystic carcinoma of the breast: case report and review of the literature. Int Semin Surg Oncol. 2006;3:17.

2. Geschickter CF, Copeland MM. Diseases of the breast: diagnosis, pathology, and treatment. Philadelphia: J. B. Lippincott; 1945.

3. Li N, Xu L, Zhao H, El-Naggar AK, Sturgis EM. A comparison of the demographics, clinical features, and survival of patients with adenoid cystic carcinoma of major and minor salivary glands versus less common sites within the Surveillance, Epidemiology, and End Results registry. Cancer. 2012;118: 3945-53.

4. Millar BA, Kerba M, Youngson B, Lockwood GA, Liu FF. The potential role of breast conservation surgery and adjuvant breast radiation for adenoid cystic carcinoma of the breast. Breast Cancer Res Treat. 2004;87:225-32.

5. Boujelbene N, Khabir A, Boujelbene N, Jeanneret Sozzi W, Mirimanoff RO, Khanfir K. Clinical review: breast adenoid cystic carcinoma. Breast. 2012;21:124-7.

6. Youk JH, Kim MJ, Kim EK, Lee JY, Oh KK, Park BW. Recurrence of adenoid cystic carcinoma in the breast after lumpectomy and adjuvant therapy. J Ultrasound Med. 2006;25:921-4.

7. Ghabach B, Anderson WF, Curtis RE, Huycke MM, Lavigne JA, Dores GM. Adenoid cystic carcinoma of the breast in the United States (1977 to 2006): a population-based cohort study. Breast Cancer Res. 2010;12:R54.
8. McClenathan JH, de la Roza G. Adenoid cystic breast cancer. Am J Surg. 2002;183:646-9.

9. Sheen-Chen SM, Eng HL, Chen WJ, Cheng YF, Ko SF. Adenoid cystic carcinoma of the breast: truly uncommon or easily overlooked? Anticancer Res. 2005;25:455-8.

10. Sumpio BE, Jennings TA, Merino MJ, Sullivan PD. Adenoid cystic carcinoma of the breast. Data from the Connecticut Tumor Registry and a review of the literature. Ann Surg. 1987;205:295-301.

11. Wetterskog D, Lopez-Garcia MA, Lambros MB, A'Hern R, Geyer FC, Milanezi F, et al. Adenoid cystic carcinomas constitute a genomically distinct subgroup of triple-negative and basal-like breast cancers. J Pathol. 2012;226:84-96.

12. Pastolero G, Hanna W, Zbieranowski I, Kahn HJ. Proliferative activity and 553 expression in adenoid cystic carcinoma of the breast. Mod Pathol. 1996;9:215-9.

13. Avery CM, Moody AB, McKinna FE, Taylor J, Henk JM, Langdon JD. Combined treatment of adenoid cystic carcinoma of the salivary glands. Int J Oral Maxillofac Surg. 2000;29:2779.

14. Leeming R, Jenkins M, Mendelsohn G. Adenoid cystic carcinoma of the breast. Arch Surg. 1992;127:233-5.

15. Coates JM, Martinez SR, Bold RJ, Chen SL. Adjuvant radiation therapy is associated with improved survival for adenoid cystic carcinoma of the breast. J Surg Oncol. 2010;102:342-7. 